Z. klin. Chem. u. klin. Biochem.

7. Jg., S. 474-479, September 1969

\title{
Enzyme des Steroid-Stoffwechsels im Blut des Menschen
}

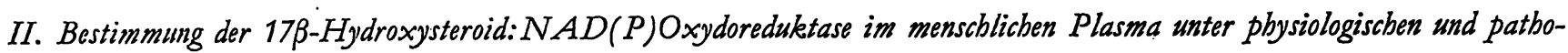 \\ logischen Bedingungen ${ }^{1}$ )
}

Von J. Breuer, V. Patt und H. Breuer

Aus dem Institut für Klinische Biochemie der Universität Bonn

(Eingegangen am 3. Juni 1969)

Im Plasma der schwangeren Frau befindet sich eine 17 $\beta$-Hydroxysteroid:NAD(P)-Oxydoreduktase, zu deren Bestimmung optimale Bedingungen ausgearbeitet wurden. Die standardisierte Methode umfaßt im wesentlichen folgende Schritte: 1. Blutentnahme unter Zusatz von Heparin, 2. Gewinnung von Plasma, 3. Inkubation von 17/-Östradiol-[6.7- $\left.{ }^{3} \mathrm{H}\right]$ mit Plasma und NAD ${ }^{\oplus}$ in Tris-Puffer, 4. Extraktion der Inkubationslösung mit Äther/Chloroform, 5. Dünnschichtchromatographie des Extraktrückstandes und 6. Messung der radioaktiven Zonen im Flüssigkeits-Szintillations-Spektrometer. An Hand von 60 Plasmauntersuchungen bei 60 verschiedenen Schwangeren wurde durch Bestimmung der Oxydation von 17 $\beta$-Östradiol zu Östron der Normalbereich der Aktivität der 17 $\beta$-Hydroxysteroid-Oxydoreduktase während der Schwangerschaft ermittelt. Die Aktivität des Enzyms nahm vom 3. Monat $(2,5 \mu \mathrm{U} / \mathrm{m} l)$ bis zum 10. Monat $(25 \mu \mathrm{U} / \mathrm{ml})$ um das 10 fache zu.

Erniedrigungen der Enzymaktivität fanden sich bei länger zurückliegenden Noxen, bei denen offenbar größere Anteile der Placenta zugrunde gegangen waten (missed abortion, Zustand nach Blutung in der Frühgravidität). Erhöhungen der Enzymaktivität wurden bei akuten Schädigungen des Placentagewebes (Abortus imminens, Übertragung, Fruchttod bei Rh-Inkompatibilität) beobachtet. Bei intaktem Trophoblasten lagen die Enzymwerte im Normalbereich. Außerhalb der Gravidität war die 17ß-Hydroxysteroid-Oxydoreduktase im Plasma einer Patientin mit Placentaresten sowie bei einem Patienten mit metastasierendem Hodenteratom nachweisbar.

\section{Enzymes of steroid metabolism in buman blood \\ II. Determination of 17ß-bydroxysteroid: $N A D(P)$-oxidoreductase in buman plasma under physiological and patbological conditions}

A method is described for the estimation of $17 \beta$-hydroxysteroid:NAD(P) oxidoreductase in the plasma of pregnant women. The standardized method consists of the following steps: 1 . collection of blood in the presence of heparin, 2 . separation of plasma, 3. incubation of $17 \beta$-oestradiol- $\left[6,7_{-}{ }^{3} \mathrm{H}\right]$ with plasma and $\mathrm{NAD}^{\odot}$ in Trisbuffer, 4. extraction of the incubation mixture with ether/chloroform, 5 . thin layer chromatography of the residue of the incubation extract and 6 . elution of the zones containing the radioactivity, and counting the radioactivity in a liquid scintillation spectrometer. The activity of the $17 \beta$-hydroxysteroid oxidoreductase was assayed in the plasma of 60 pregnant women by measuring the rate of oxidation of $17 \beta$-oestradiol to oestrone. No activity of the enzyme could be demonstrated with certainty during the first two months of pregnancy. A 10-fold increase in the activity of the enzyme was observed from the third month $(2.5 \mu \mathrm{U} / \mathrm{m} l)$ up to the tenth month $(25 \mu \mathrm{U} / \mathrm{m} l)$.

The activity of the $17 \beta$-hydroxysteroid oxidoreductase was decreased in subjects with a history of damaged placenta (abortion after bleeding in early pregnancy), whereas an increased activity was found in patients with acute damage of the placenta (abortus imminens, postmaturity, foetal death due to $\mathrm{Rh}$-incompatibility). The values were within the normal range in subjects with intact trophoblasts. $17 \beta$ hydroxysteroid oxidoreductase activity could be detected in a patient having placenta vestiges and in a patient with a metastatizing tumour of the testis.

Im Serum der schwangeren Frau findet sich eine 17 $\beta$-Hydroxysteroid:NAD(P)-Oxydoreduktase $\left.{ }^{2}\right) \quad(\mathrm{EC}$ 1.1.1. 62), die nur mit phenolischen (z. B. 17 $\beta$-Östradiol), nicht aber mit neutralen Steroiden (z. B. Testosteron) reagiert und durch Fällung mit Ammoniumsulfat angereichert werden kann (2). Die Ergebnisse kinetischer Untersuchungen sowie die Tatsache, daß die 17 $\beta$ Hydroxysteroid-Oxydoreduktase bislang ausschließlich bei Schwangeren nachgewiesen wurde, sprechen für einen plazentaren Ursprung des Enzyms (2).

Im Hinblick auf die mögliche Bedeutung der $17 \beta-$ Hydroxysteroid-Oxydoreduktase für die klinisch-chemische Diagnostik erschien es wünschenswert, die optimalen Bedingungen für die Aktivitätsbestimmung

1) Auszugsweise vorgetragen von V. P. deutung von Radioisotopen in der Klinischen Chemie und Klinischen Biochemie", 6. - 8. 12. 1968 in Köln (1):

2) Im folgenden als $17 \beta$-Hydroxysteroid-Oxydoreduktase bezeichnet. festzulegen. In der vorliegenden Arbeit wird an Hand von 60 Einzelbestimmungen der Normalbereich der Enzymaktivität für die Zeit vom 3. bis 10. Monat der Schwangerschaft angegeben; außerdem wird über die Aktivität der $17 \beta$-Hydroxysteroid-Oxydoreduktase unter pathologischen Bedingungen während und außerhalb der Schwangerschaft berichtet. Die Bestimmung der Enzymaktivität erfolgte in allen Fällen durch Messung der Oxydation von 17 $\beta$-Östradiol zu Östron bei einem $\mathrm{pH}$-Wert von 9,0 .

\section{Methodik}

Steroide

Ostron-[6.7- $\left.{ }^{3} \mathrm{H}\right]$ (3-Hydroxyöstra-1.3.5(10)-trien-17-on-[6.7- $\left.{ }^{3} \mathrm{H}\right]$; spez. Aktivität $128 \mathrm{mC} / \mathrm{mMol}$ ) wurde durch Reduktion von 6-Dehydro-östron (3-Hydroxyöstra-1.3.5(10).6-tetraen-17-on) mit Tritium gewonnen. 17 $\beta$-Östradiol-[6.7-3 $\mathrm{H}]$ (Östra-1.3.5(10)-trien$3.17 \beta$-diol-[6.7-3 H]; spez. Aktivität $128 \mathrm{mC} / \mathrm{mMol}$ ) wurde durch Reduktion von Ostron-[6.7-3 $\mathrm{H}]$ mit Natriumboranat dargestellt. 
Die radioaktiven Steroide wurden vor den Versuchen papierchromatographisch auf Reinheit geprüft.

\section{Reagenzien, Lösungsmittel und Puffer}

Mit Ausnahme von Folin-Ciocalteu's Reagenz (3) waren alle Reagenzien von p. a. Reinheitsgrad (E. Merck, Darmstadt). Die organischen Lösungsmittel wurden vor Gebrauch destilliert. $\mathrm{NAD}^{\oplus}$ wurde von Biochemica Boehringer, Mannheim, bezogen. Als Puffer wurde 0,2M Tris-HCl-Puffer ( $\mathrm{pH} 9,0$ ) verwendet.

Gewinnung von Plasma und Serum

Zur Gewinnung von Plasma wurde venöses Blut mit Heparin (20 I. E. $/ \mathrm{ml}$ Blut) versetzt und sofort $5 \mathrm{Min}$. bei $2000 \mathrm{~g}$ zentrifugiert. Serum wurde durch Zentrifugation (5 Min. bei $2000 \mathrm{~g}$ ) von venösem Blut gewonnen.

\section{Inkubationsbedingungen}

Alle Versuche wurden bei $40^{\circ}$ für $60 \mathrm{Min}$. in einem Schüttelthermostaten unter Luft durchgeführt. Jeweils $3,7 \mathrm{nMol}(0,47 \mu \mathrm{C})$ $17 \beta-$ Ostradiol- $\left[6.7-{ }^{3} \mathrm{H}\right]$, gelöst in $0,02 \mathrm{~m} l$ Propylenglykol, wurden mit $1,0 \mathrm{ml}$ Plasma oder Serum in Gegenwart von $4,5 \mu \mathrm{Mol} \mathrm{NAD}^{\oplus}$ und $2,0 \mathrm{~m} / 0,2 \mathrm{M}$ Tris-HCl-Puffer ( $\mathrm{pH} 9,0$ ) inkubiert.

\section{Aufarbeitung der Versuchsansätze}

Nach Zusatz von je $74 \mathrm{nMol}(20 \mu \mathrm{g})$ Östron und $17 \beta$-Östradiol wurden die Inkubationslösungen dreimal mit je $10 \mathrm{ml}$ Åther/ Chloroform $(3: 1 \mathrm{v} / \mathrm{v})$ extrahiert, die Extrakte vereinigt und bei $35^{\circ}$ unter Stickstoff eingedampft. Die Extraktrückstände wurden in etwa $0,5 \mathrm{ml}$ Athanol aufgenommen.

\section{Dünnscbicbtchromatograpbie}

In allen Versuchen wurde Kieselgel $\mathrm{HF}_{254}$ (E. Merck, Darmstadt) als Trägermittel verwendet. Die Trennung der Substanzen erfolgte im System Benzol/Athylacetat $(2: 1 \mathrm{v} / \mathrm{v})$ durch zweimalige Entwicklung der Chromatogramme. Bei einer Laufzeit von $2 \times 20 \mathrm{Min}$. betrugen die Wanderungsstrecken für Östron etwa $12,5 \mathrm{~cm}$ und für $17 \beta$-Ostradiol etwa $8,5 \mathrm{~cm}$. Die Lokalisierung der nachzuwèisenden Ostrogene (17 $\beta$-Östradiol und Ostron) erfolgte durch Vergleich mit den Wanderungsstrecken der authentischen, nichtradioaktiven Referenzsubstanzen, die mit Folin-Ciocalteu's Reagenz (3) sichtbar gemacht wurden.

\section{Quantitative Bestimmungen}

Nach ihrer Lokalisierung durch die Referenzsubstanzen wurden die nichtangefärbten Positionen (radioaktives Ostron und $17 \beta$ Ostradiol) mit einer Rasierklinge abgekratzt. Nach dem Uberführen in ein Zentrifugenglas wurde das Kieselgel mit $3 \mathrm{ml}$ Methanol überschichtet und durch Aufwirbeln mit einem Spatel im organischen Lösungsmittel suspendiert; anschließend wurde die Suspension für $10 \mathrm{Min}$. bei $1500 \mathrm{~g}$ zentrifugiert und der Überstand dekantiert. Dieser Elutionsvorgang wurde insgesamt dreimal wiederholt. Die methanolischen Exxtrakte (etwa $9 \mathrm{~m} l$ ) wurden in Tricarb-Zählgefäße (Fa. Packard, Frankfurt/M.) überführt und unter einer Infrarot-Lampe zur Trockene eingedampft. Die Rückstände wurden in $12 \mathrm{ml}$ einer Szintillations-Lösung, enthaltend $5 \mathrm{~g} / \mathrm{l}$ 2.5-Diphenyloxazol (PPO) und 0,3 g/l 1.4-Bis-[4-methyl-5phenyl-oxazolyl-(2)]-benzol (Dimethyl-POPOP), unter Verwendung eines externen Standards in einem Packard Tri-Carb-Szintillations-Spektrometer (Modell 3003) gemessen. Die Zählausbeute für ${ }^{3} \mathrm{H}$ betrug $43 \%$.

\section{Enzymaktivität}

$\mathrm{Da}$ die Aktivität der 17 $\beta$-Hydroxysteroid-Oxydoreduktase vergleichsweise gering ist, wird die Enzymkonzentration nicht - wie üblich — in $\mathrm{mU} / \mathrm{ml}$, sondern in $\mu \mathrm{U} / \mathrm{m} l$ angegeben.

\section{Ergebnisse}

Untersuchungen zur Stabilität der 17ß-Hydroxysteroid-Oxydoreduktase

In früheren Versuchen (2) hatte sich gezeigt, daß die Aktivität der 17. $\beta$-Hydroxysteroid-Oxydoreduktase im

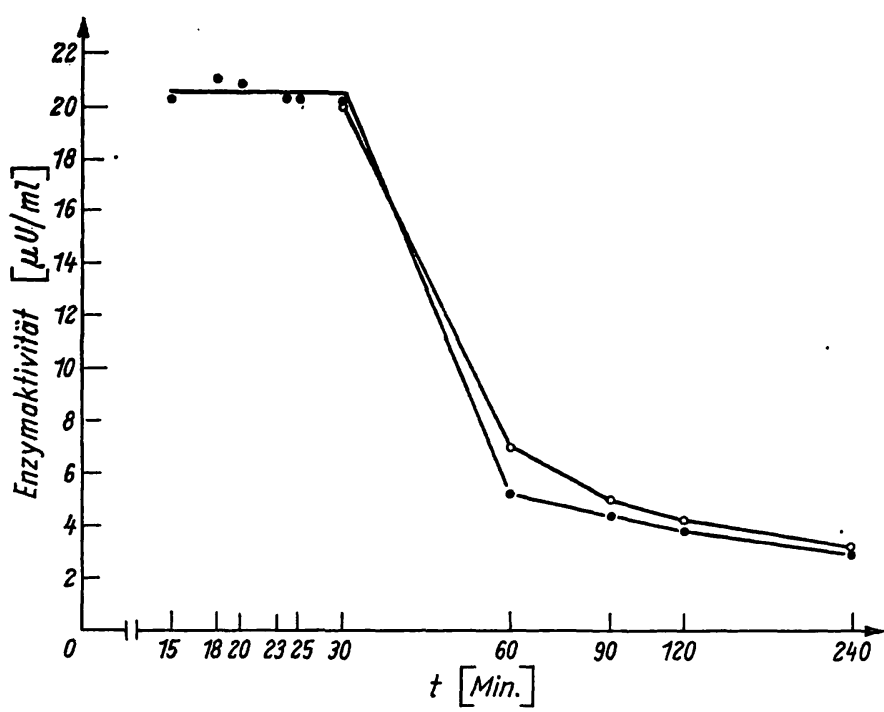

Abb. 1

Verhalten der Aktivität der $17 \beta$-Hydroxysteroid-Oxydoreduktase in Abhängigkeit von der Zeit zwischen Blutentnahme und Inkubationsbeginn. Jeweils $3,7 \mathrm{nMol} 17 \beta-O$ stradiol- $\left[6.7--^{2} \mathrm{H}\right]$ wurden mit $1,0 \mathrm{ml}$ Plasma (०. bzw. $1,0 \mathrm{ml}$ Serum (o von 4,5 $\mu$ Mol $\mathrm{NAD}{ }^{\oplus}$ und $2,0 \mathrm{ml} 0,2 \mathrm{M}$ Tris-HCl-Puffer $(\mathrm{pH} 9,0)$ für 1 Std. bei $40^{\circ}$ inkubiert. Die Punkte der Kurven sind Mittelwerte aus 2 Einzelbestimmungen. Plasma und Serum wurden aus einer Blutprobe (37. Schwangerschaftswoche) gewonnen

Serum sowohl bei Raumtemperatur als auch bei $+5^{\circ}$ und $-18^{\circ}$ deutlich abnimmt. Bei der Ausarbeitung optimaler Bedingungen war es deshalb notwendig, den zeitlichen Verlauf dieses Aktivitätsverlustes genauer zu untersuchen. Wie aus Abbildung 1 hervorgeht, bleibt die Enzymaktivität im Plasma von der 15.-30. Min. nach Entnahme des Blutes praktisch konstant. Anschließend erfolgt ein rascher Abfall, der sowohl im Plasma als auch im Serum beobachtet wird. Nach 2stdg. Aufbewahrung des Plasmas oder Serums bei $20^{\circ}$ hat die Enzymaktivität gegenüber dem Ausgangswert um etwa $75 \%$ abgenommen. Der in Abbildung 1 dargestellte Verlauf der Aktivitätsabnahme konnte an 3 weiteren Plasmaproben reproduziert werden.

\section{Aktivität der 17ß-Hydroxysteroid-Oxydoreduktase im Plasma und im Serum}

Wie bereits aus Abbildung 1 ersichtlich, weist die Enzymaktivität für den Zeitraum von 30 bis 240 Min. nach Entnahme des Blutes keine wesentlichen Unterschiede zwischen Plasma und Serum auf. Um diese Frage eingehender zu prüfen, wurden in vergleichenden Untersuchungen die Aktivität der $17 \beta$-HydroxysteroidOxydoreduktase bei 10 Schwangeren im Plasma und im Serum ermittelt (Tab. 1). Die Inkubationen erfolgten 30 Min. nach Blutentnahme. Innerhalb der methodischen Fehlergrenzen waren die Werte im Plasma und im Serum identisch. Im Hinblick auf die nach $30 \mathrm{Min}$. rasch einsetzende Inaktivierung des Enzyms wurde der Beginn der Inkubationen auf genau 20 Min. nach Blutentnahme festgelegt. $\mathrm{Da}$ die Gewinnung von Plasma einen geringeren Zeitaufwand erfordert als diejenige von Serum, wurde aus Gründen der Praktikabilität für alle weiteren Aktivitätsbestimmungen Plasma verwendet. 
Tab. 1

Aktivität der $17 \beta$-Hydroxysteroid-Oxydoreduktase im Plasma und im Serum derselben Blutprobe. Jeweils $3,7 \mathrm{nMol} 17 \beta$-O stradiol- $\left[6.7^{-2} \mathrm{H}\right]$ wurden mit $1,0 \mathrm{ml}$ Plasma bzw. $1,0 \mathrm{ml}$ Serum in Gegenwart von $4,5 \mu \mathrm{Mol} N A D^{\oplus}$ und $2,0 \mathrm{ml} 0,2 \mathrm{M}$ Tris-HCl-Puffer $(\mathrm{pH} \mathrm{9,0)}$ für 1 Std. bei $40^{\circ}$ inkubiert. Die Zeit zwischen Blutentnahme und Inkubationsbeginn betrug 30 Min. Alle Werte sind Mittelwerte aus 3 Einzelbestimmungen

\begin{tabular}{|c|c|c|c|}
\hline Versuch & Schwangerschaftswoche & $\begin{array}{l}\text { Enzyma } \\
\text { Plasma }\end{array}$ & $\begin{array}{l}\mu \mathrm{U} / \mathrm{ml} \\
\text { Serum }\end{array}$ \\
\hline $\begin{array}{l}1 \\
2 \\
3 \\
4 \\
5 \\
6 \\
7 \\
8 \\
9\end{array}$ & $\begin{array}{r}7 . \\
9 . \\
9 . \\
9 . \\
9 . \\
11 . \\
12 . \\
12 . \\
12 .\end{array}$ & $\begin{array}{l}0,4 \\
2,0 \\
3,1 \\
3,6 \\
4,9 \\
5,4 \\
2,4 \\
5,9 \\
6,3\end{array}$ & $\begin{array}{l}0,4 \\
2,1 \\
2,8 \\
3,5 \\
4,8 \\
5,5 \\
2,7 \\
6,0 \\
6,1\end{array}$ \\
\hline
\end{tabular}

Optimale Bedingungen qur Bestimmung der Aktivität der 17ß-Hydroxysteroid-Oxydoreduktase

Auf Grund der vorangegangenen Versuche wurden in Abwandlung der früher beschriebenen Methodik (2) folgende optimale Bedingungen festgelegt:

1. Gewinnung von Plasma nach Zusatz von 20 I.E. Heparin/ml Blut.

2. Inkubation von $1 \mathrm{~m} l$ Plasma mit $17 \beta$-Östradiol$\left(6.7-{ }^{3} \mathrm{H}\right)$ unter den in der Methodik angegebenen Bedingungen. Inkubationsbeginn genau $20 \mathrm{Min}$. nach Blutentnahme; Inkubationsdauer $60 \mathrm{Min}$.

3. Extraktion der Inkubationslösung mit Äther/Chloroform $(3: 1 \mathrm{v} / \mathrm{v} ; 3 \times 10 \mathrm{~m})$.

4. Zweimalige Dünnschichtchromatographie des Extraktrückstandes auf Kieselgel im System Benzol/ Äthylacetat (2:1) (weitere Einzelheiten vgl. Methodik); durch Verwendung der Dünnschichtchromatographie an Stelle der Papierchromatographie ergibt sich ein Zeitgewion von etwa 3 Stdn.

5. Elution der radioaktiven Zonen von den Dünnschichtchromatogrammen und quantitative Bestimmung von $17 \beta$-Östradiol- $\left[6.7-{ }^{3} \mathrm{H}\right]$ und Östron- $\left[6.7-{ }^{3} \mathrm{H}\right]$ im Szintillations-Spektrometer.

Die mittlere Gesamtwiederfindung [17 $\beta$-Östradiol $\left(\mathrm{E}_{2}\right)$ und Östron $\left(\mathrm{E}_{1}\right)$ ] beträgt bei dem hier beschriebenen Verfahren etwa $80 \%$. Zur Bestimmung der A.ktivität der $17 \beta$-Hydroxysteroid-Oxydoreduktase ist es notwendig, auf $100 \%$ Wiederfindung zu korrigieren. Mit Hilfe folgender Berechnung wird die tatsächlich umgesetzte Menge $17 \beta$-Östradiol festgestellt (alle Werte in $\mathrm{nMol}$ ):

$E_{2}$ umgesetzt $=\frac{E_{1} \text { gebildet }}{E_{2} \text { wiedergefunden }+E_{1} \text { gebildet }} \times E_{2}$ eingesetzt

Die Präzision wurde durch eine 10fach-Bestimmung in einer Plasmaprobe (36. Schwangerschaftswoche) ermittelt. Das Ergebnis ist in Abbildung 2 dargestellt. Bei einem Mittelwert von $21,8 \mu \mathrm{U} / \mathrm{m} l$ betrug die Standardabweichung $(S) \pm 0,86$ und der Variationskoeffizient (VK) $4 \%$.

Bestimmung des Normalbereiches der 17ß-HydroxysteroidOxydoreduktase wäbrend der Schwangerschaft

Alle Plasmaproben stammten von Probandinnen, bei denen der Schwangerschaftsverlauf ungestört blieb. Zur

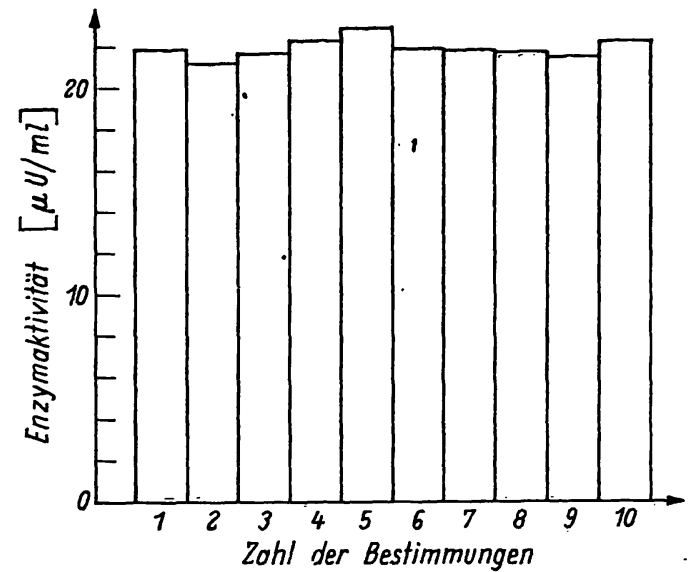

Abb. 2

Graphische Darstellung einer 10 fach-Bestimmung der Aktivität der $17 \beta$-Hydroxysteroid-Oxydoreduktase in einer Plasmaprobe (36.Schwangerschaftswoche). Jeweils $3,7 \mathrm{nMol} 17 \beta-O$ stradiol- $[6.7-2 \mathrm{H}]$ wurden mit $1,0 \mathrm{ml}$ Plasma in Gegenwart von 4,5 $\mu \mathrm{Mol} \mathrm{NAD}^{\odot}$ und $2,0 \mathrm{ml} 0,2 \mathrm{M}$ ris- $\mathrm{HCl}$-Puffer $\left(\mathrm{pH} \mathrm{9,0)}\right.$ für 1 Std. bei $40^{\circ}$ inkubiert. Die Zeit zwischen Blutentnahme und Inkubationsbeginn betrug 20 Min. Alle Werte sind Mittelwerte aus 3 Einzelbestimmungen

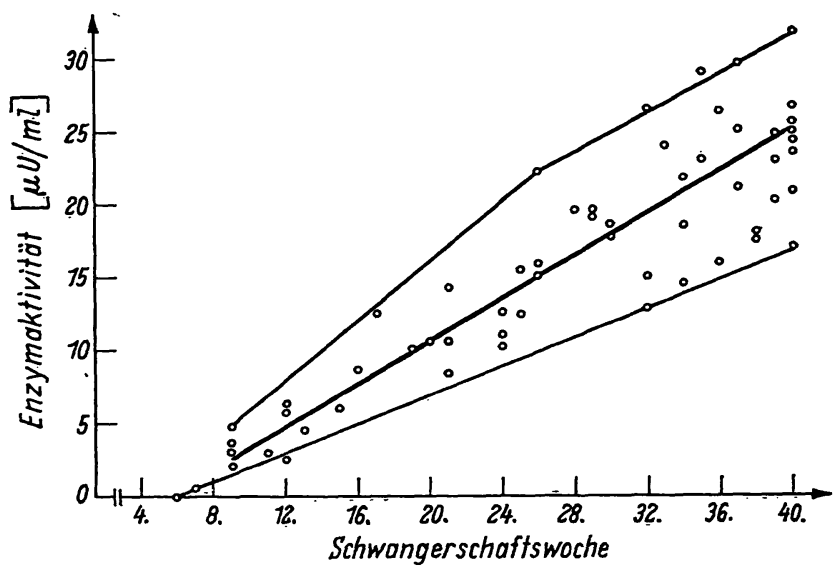

Abb. 3

Normalbereich der Aktivität der 17 $\beta$-Hydroxysteroid-Oxydoreduktase im Plasma in Abhängigkeit von der Schwangerschaftswoche. Jeweils $3,7 \mathrm{nMol} 17 \beta-$ - $\mathrm{s}$ tradiol- $\left[6 .-^{2} \mathrm{H}\right]$ wurden mit $1,0 \mathrm{ml}$ Plasma in Gegenwart von 4,5 $\mu$ Mol $\mathrm{NAD}^{\oplus}$ und $2,0 \mathrm{ml} 0,2 \mathrm{M}$ Tris-HCl-Puffer $(\mathrm{pH} \mathrm{9,0})$ Inkubationsbeginn betrug $20 \mathrm{Min}$. Alle Werte sind Mittelwerte aus 3 Einzelbestimmungen

Festlegung des Normalbereiches wurden 60 Plasmaproben von $60 \mathrm{Schwangeren} \mathrm{in} \mathrm{der} \mathrm{Zeit} \mathrm{vom} \mathrm{3.} \mathrm{bis} \mathrm{zum}$ 10. Schwangerschaftsmonat untersucht. Abbildung 3 zeigt das Verhalten der Enzymaktivitāten während der Schwangerschaft. Bei Verwendung von $1 \mathrm{~m} l$ Plasma ist die Aktivitāt der 17 $\beta$-Hydroxysteroid-Oxydoreduktase zu Beginn des 3. Schwangerschaftsmonats mit Sicherheit nachweisbar; die höchsten Werte werden unmittelbar ante partum erreicht. Die durchschnittliche Aktivität der 17 $\beta$-Hydroxysteroid-Oxydoreduktase nimmt vom Beginn des 3. Schwangerschaftsmonates $(2,5 \mu \mathrm{U} / \mathrm{m} l)$ bis zum Ende der Schwangerschaft $(25 \mu \mathrm{U} / \mathrm{ml})$ um das 10 fache zu.

Unter den ungestört verlaufenden Schwangerschaften nimmt die Zwillingsgravidität eine Sonderstellung ein. Bei einer Frau, die in der 33. Schwangerschaftswoche von zwei Mädchen mit einem Geburtsgewicht von 1400 und $1750 \mathrm{~g}$ entbunden wuide, betrug die Aktivität der 17k-Hydroxysteroid-Oxydoreduktase in der 24. Woche $19,4 \mu \mathrm{U} / \mathrm{m} l$ (Mittelwert des Normalbereichs 
Tab. 2

Aktivität der 17 1 -Hydroxysteroid-Oxydoreduktase im Plasma unter pathologischen Bedingungen während der Schwangerschaft. Jeweils $3,7 \mathrm{nMol} 17 \beta-O$ stradiol-[6.7-'H] wurden mit $1,0 \mathrm{ml}$ Plasma in Gegenwart von 4,5 $\mu \mathrm{Mol}$ NAD ${ }^{\oplus}$ und $2,0 \mathrm{ml} 0,2 \mathrm{M}$ Tris-HCl-Puffer (pH 9,0) für

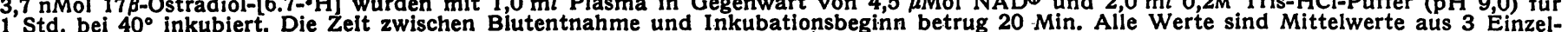
Std. bei $40^{\circ}$ inkubiert. Die Zelt zwischen Blutentnahme und $\begin{gathered}\text { Inkubationsbe } \\ \text { bestimmungen }\end{gathered}$

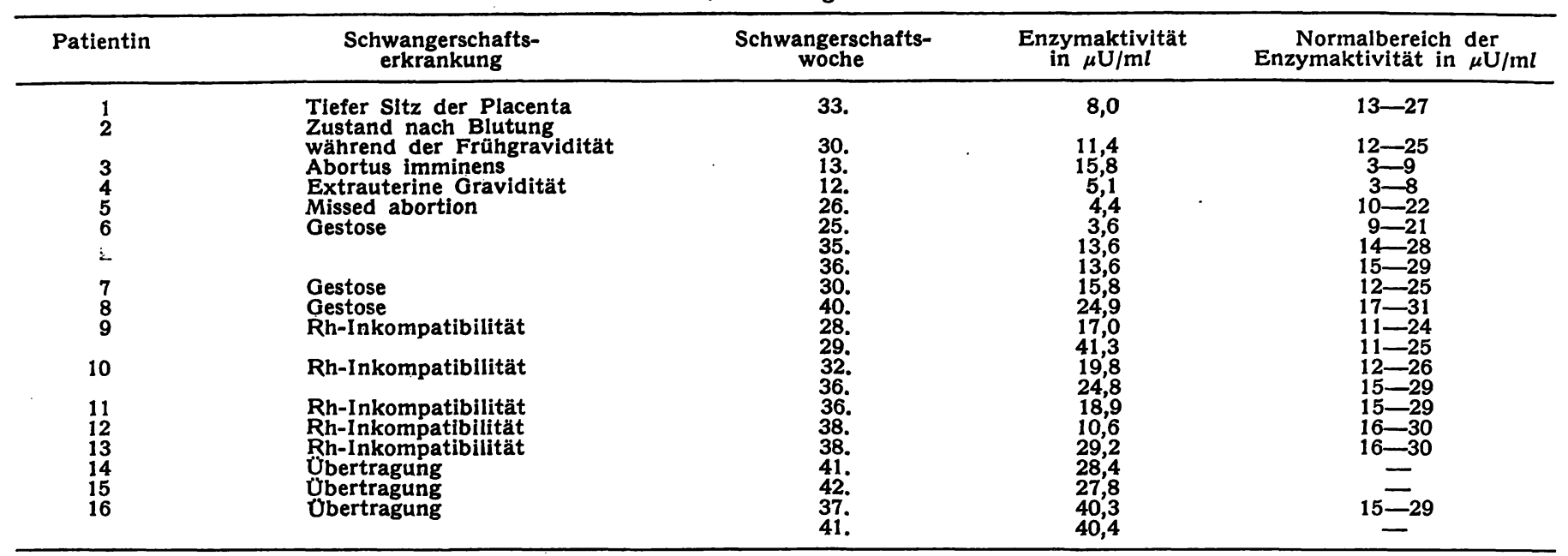

Tab. 3

Aktivität der $17 \beta$-Hydroxysteroid-Oxydoreduktase im Plasma unter pathologischen Bedingungen außerhalb der Schwangerschaft. Jeweils

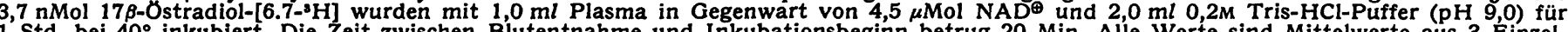
1 Std. bei $40^{\circ}$ inkubiert. Die Zeit zwischen Blutentnahme und Inkubationsbeginn betrug 20 Min. Alle Werte sind Mittelwerte aus 3 Einzelbestimmungen. AZR = Aschheim-Zondek-Reaktion; IST = Immunologischer Schwangerschaftstest

\begin{tabular}{|c|c|c|c|c|}
\hline Patient & Geschlecht & Erkrankung & $\begin{array}{c}\text { Enzymaktivität } \\
\text { in } \mu \mathrm{U} / \mathrm{ml}\end{array}$ & Bemerkungen \\
\hline $\begin{array}{l}1 \\
2 \\
3 \\
1 .\end{array}$ & 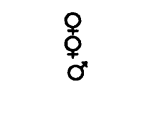 & $\begin{array}{l}\text { Retention von Placentaresten } \\
\text { Destruierende Blasenmole } \\
\text { Teratom des Hodens }\end{array}$ & $\begin{array}{r}4,7 \\
<0,5 \\
7,6\end{array}$ & $\begin{array}{l}20 \text { Tage post partum } \\
\text { Behandlung mit Methotrexat } \\
\text { Histologisch gesichert; } \\
\text { keine Behandlung mit Methotrexat; } \\
\text { AZR und IST positiv }\end{array}$ \\
\hline $\begin{array}{l}4 \\
5 \\
6\end{array}$ & $\begin{array}{l}\sigma^{\prime \prime} \\
\sigma^{\prime \prime} \\
\sigma^{\prime \prime}\end{array}$ & $\begin{array}{l}\text { Chorionepitheliom } \\
\text { Chorionepitheliom } \\
\text { Chorionepitheliom }\end{array}$ & $\left.\begin{array}{l}<0,5 \\
<0,5 \\
<0,5\end{array}\right\}$ & $\begin{array}{l}\text { Histologisch gesichert; } \\
\text { Behandlung mit Methotrexat; } \\
\text { AZR und IST positiv }\end{array}$ \\
\hline
\end{tabular}

$13,5 \mu \mathrm{U} / \mathrm{ml}$ ) und in der 33. Woche 33,6 $\mu \mathrm{U} / \mathrm{ml}$ (Mittelwert des Normalbereichs $20,0 \mu \mathrm{U} / \mathrm{m} l)$. Während der erste Wert an der obersten Grenze der Norm lag, war der zweite Wert deutlich erhöht.

Verbalten der 17ß-Hydroxysteroid-Oxydoreduktase unter pathologischen Bedingungen wäbrend der Sscbwangerschaft

Die Aktivität der $17 \beta$-Hydroxysteroid-Oxydoreduktase zeigte bei einigen Schwangerschaftserkrankungen wesentliche Abweichungen vom Normalbereich (Tab. 2). So fanden sich deutliche Erniedrigungen bei einem tiefen Sitz der Placenta (Pat. 1), bei einem Fall von missed abortion (Pat. 5), bei einer Gestose (Pat. 6) sowie bei einer Schwangeren mit Rh-Inkompatibilität (Pat. 12). Deutliche Erhöhungen der Enzymaktivität wurden bei einem Abortus imminens (Pat. 3), einer Rh-Inkompatibilität (Pat. 9) und im Falle einer Übẹttragung (Pat. 16) beobachtet. Bei den übrigen 10 Patientinnen mit Schwangerschaftserkrankungen ergaben sich keine Abweichungen vom Normalbereich.

Bei einer diabetischen Schwangeren war zwischen der 27. und 36. Schwangerschaftswoche eine Verlaufsbeobachtung möglich (Abb.4). Die Aktivität der 17 Hydroxysteroid-Oxydoreduktase bewegte sich - mit Ausnahme eines Wertes - im Normalbereich; es sei erwähnt, daß eine Schädigung der Frucht nicht vorlag. Drei weitere Einzelbeobachtungen bei diabetischen Schwangeren ergaben für die $1.7 \beta$-Hydroxysteroid-

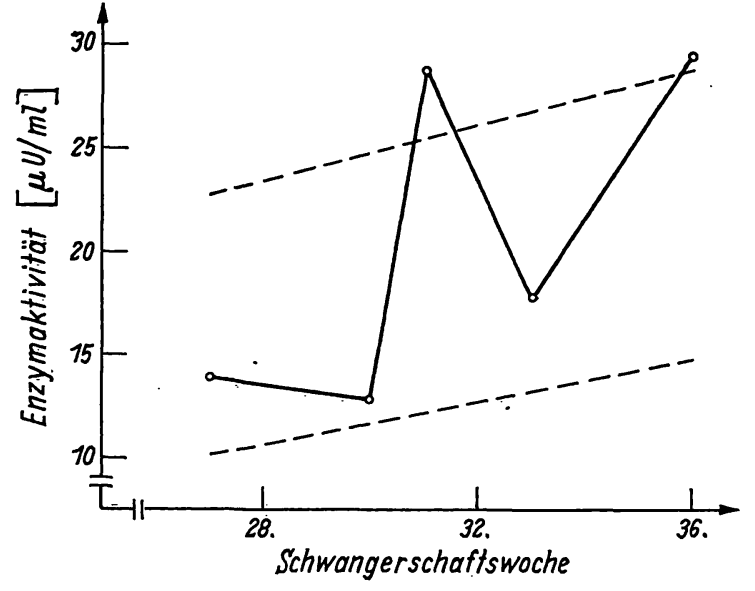

Abb. 4

Verhalten der Aktivität der 17 $\beta$-Hydroxysteroid-Oxydoreduktase im Plasma einer diabetischen Schwangeren. Jeweils 3,7 nMol $17 \beta-O$ stra-

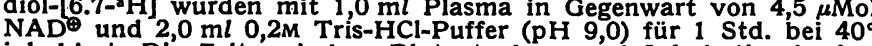
inkubiert. Die Zeit zwischen Blutentnahme und Inkubationsbeginn betrug 20 Min. Alle Werte sind Mittelwerte aus 3 Einzelbestimmungen Die oberen und unteren Grenzen des Normalbereichs sind durch gestrichelte Linien markiert

Oxydoreduktase ebenfalls Werte im Normalbereich. Die Patientinnen standen unter Insulintherapie; auch diese Schwangerschaften verliefen - abgesehen von der Stoffwechselschädigung - ohne Komplikationen.

$V$ erbalten der 17ß-Hydroxysteroid-Oxydoreduktase unter pathologischen Bedingungen außerbalb der Schwangerschaft

Die Ergebnisse dieser Untersuchungen sind in Tabelle 3 zusammengestellt. Bei einer Retention von Placenta- 
resten (Pat. 1) sowie bei einem cytostatisch noch nicht behandelten Teratom des Hodens (Pat. 3) war eine Aktivität der $17 \beta$-Hydroxysteroid-Oxydoreduktase im Plasma nachweisbar. Im Gegensatz dazu konnte im Falle einer destruierenden Blasenmole (Pat. 12) sowie bei drei Chorionepitheliomen des Mannes (Pat. 4, 5 und 6) keine Aktivität mit Sicherheit nachgewiesen werden; diese Patienten standen unter Behandlung mit hohen Dosen eines Antimetaboliten (Methotrexat).

\section{Diskussion}

Ein wesentliches Ziel der vorliegenden Untersuchungen war die Festlegung optimaler Bedingungen zur Aktivitätsbestimmung der 17 $\beta$-Hydroxysteroid-Oxydoreduktase im Blut der schwangeren Frau. Die Ausarbeitung einer für die klinisch-chemische Diagnostik brauchbaren Methode wurde durch die Tatsache erschwert, daß die 17ß-Hydroxysteroid-Oxydoreduktase ein sehr instabiles Enzym ist. Es zeigte sich, daß bereits $30 \mathrm{Min}$. nach Entnahme des Blutes ein starker Abfall der Aktivität einsetzt; demnach ist es notwendig, die Aktivitätsbestimmungen möglichst rasch vorzunehmen. $\mathrm{Da}$ einerseits die Gewinnung von Serum längere Zeit in Anspruch nimmt als diejenige von Plasma, andererseits keine Aktivitätsunterschiede zwischen Serum und Plasma erkennbar sind, erwies es sich als zweckmäßig, alle Enzymbestimmungen im Plasma durchzuführen. Damit ist es möglich, ohne Schwierigkeiten den Test zu einem Zeitpunkt (20 Min. nach Blutentnahme) zu beginnen, an dem die Enzymaktivität noch nicht abgenommen hat. In diesem Zusammenhang sei erwähnt, daß durch das Substrat (17 $\beta$-Östradiol) eine Stabilisierung des Enzyms erfolgt.

Die Anwendung der Dünnschichtchromatographie an Stelle der früher (2) verwendeten Papierchromatographie führte bei der Auftrennung von 17 $\beta$-Östradiol und Östron zu einer erheblichen Zeitersparnis. Eine weitere Verkürzung der Aufarbeitungsdauer kann durch Verwendung eines. Dünnschichtradiochromatographen erzielt werden.

An Hand von 60 Plasmauntersuchungen bei 60 verschiedenen Schwangeren wurde der Normalbereich der Aktivität der 17 $\beta$-Hydroxysteroid-Oxydoreduktase vom 3. bis zum 10. Schwangerschaftsmonat ermittelt. Während dieses Zeitraumes nahm die Aktivität des Enzyms um etwa das 10fache zu. Alle gemessenen Normalwerte bewegen sich in einem Bereich von \pm 30 bis $40 \%$ um den jeweiligen Mittelwert. Unter den hier gewählten optimalen Reaktionsbedingungen wurden am Ende der Schwangerschaft etwa $40 \%$ vom eingesetzten $17 \beta$-Östradiol zu Östron oxydiert.

Über die Anwesenheit von Steroidenzymen im Erythrocyten ist seit der ersten Mitteilung von WeRTHESSEN, BAKER und BORCI (4) im Jahre 1948 wiederholt berichtet worden [vgl. (5)]. Der Nachweis von Enzymen des Steroidstoffwechsels im menschlichen Serum und Plasma konnte jedoch in den folgenden zwei Jahrzehnten nicht eindeutig geführt werden. Lediglich
Brown, Golder und Wright (6) fanden bei acht Inkubationen mit menschlichem Plasma eine Oxydation von $17 \beta$-Östradiol zu Östron, deren Ausmaß 6,4\% betrug; die Autoren hielten es jedpch für wahrscheinlich, daß für diesen Umsatz ein aus den Erythrocyten freigesetztes Enzym verantwortlich sei.

Nach unseren bisherigen Untersuchungen ist es außerhalb der Schwangerschaft unter physiologischen Bedingungen nicht möglich, im Plasma einer hämolysefreien menschlichen Blutprobe die Oxydoreduktion von $17 \beta$-Östradiol und Östron nachzuweisen. Die bereits früher geäußerte Vermutung (2), wonach die Placenta als Ursprungsorgan der 17 $\beta$-Hydroxysteroid-Oxydoreduktase anzusehen sei, wird durch die Befunde dẹr vorliegenden Arbeit weiter unterstützt. In diesem $\mathrm{Zu}$ sammenhang kommt dem Nachweis einer Enzymaktivität im Falle der Retention von Placentaresten besondere Bedeutung zu. Während normalerweise $18 \mathrm{Std}$. nach der Geburt kein Umsatz von 17 $\beta$-Östradiol zu Östron im Plasma mehr stattfindet, wurde im vorliegenden Falle noch 20 Tage post partum eine Aktivität von $4,7 \mu \mathrm{U} / \mathrm{m} l$ festgestellt; die Diagnose wurde durch histologische Untersuchung des abradierten Gewebsmaterials gesichert. Aufgrund dieses Ergebnisses ist anzunehmen, da $\beta$ die Freisetzung der 17 $\beta$-Hydroxysteroid-Oxydoreduktase aus der Placenta in das Blut unabhängig von der Anwesenheit und dem Wohlbefinden des Föten erfolgt.

Ähnlich wie das Trophoblastengewebe ist auch das Chorionepitheliom und das Hodenteratom des Mannes zur Bildung von Gonadotropinen und Steroiden (7) befähigt. Es ist deshalb nicht verwunderlich, daß im Plasma eines Patienten mit unbehandeltem, metastasierenden Hodenteratom eine Aktivität der $17 \beta$-Hydroxysteroid-Oxydoreduktase von $7,6 \mu \mathrm{U} / \mathrm{m} l$ nachgewiesen werden konnte; dieser Wert wird sonst nur nach einer Schwangerschaftsdauer von mindestens 3 Monaten beobachtet. Weitere Hinweise für die Bildung der 17 $\beta$ Hydroxysteroid-Oxydoreduktase im Trophoblastengewebe ergeben sich aus dem relativ hohen Substratumsatz bei einer Zwillingsschwangerschaft und bei Nebenplacenten im Falle einer Übertragung; andererseits war die Enzymaktivität bei abgestorbener Frucht (missed abortion) deutlich erniedrigt.

Neben erhöhten und erniedrigten Werten wurden bei pathologischen Schwangerschaftsverläufen auch Normalwerte für die 17 $\beta$-Hydroxysteroid-Oxydoreduktase gemessen. Unter Berücksichtigung des klinischen Verlaufs können diese unterschiedlichen Befunde wie folgt erklärt werden:

1. Eine akute Schädigung des Placentagewebes, die mit frischen Nekrosen einhergeht, führt zu einer verstärkten Freisetzung der $17 \beta$-Hydroxysteroid-Oxydoreduktase in das Blut (Abortus imminens, Ubertragung, Fruchttod bei Rh-Inkompatibilität).

2. Sind bei einer länger zurückliegenden placentaren Noxe größere Anteile der $\mathrm{Placenta} \mathrm{zugrunde} \mathrm{gegangen,}$ so ist der Úbertritt des Enzyms von der Placenta in das 
mütterliche Blut vermindert (missed abortion, Zustand nach Blutung in der Frühgravidität).

3. Bei intaktem Trophoblasten bleiben die Enzymaktivitäten - trotz klinischer Zeichen einer Schwangerschaftserkrankung - im Normalbereich.

Bei der Diagnostik von Funktionsstörungen der foetoplacentaren Einheit wird der Bestimmung von Östriol und von Pregnandiol im Urin eine nicht unerhebliche Bedeutung zugesprochen [vgl. $(8,9)]$. Im Hinblick auf die placentare Herkunft der 17 $\beta$-Hydroxysteroid-Oxydo- reduktase im Plasma der Schwangeren erscheint es nicht ausgeschlossen, durch die gleichzeitige Bestimmung von Steroidhormonen und Enzymen des Steroidstoffwechsels weitere Einblicke in die Pathophysiologie der gestörten Schwangerschaft zu gewinnen.

Die vorliegende Untersuchung wurde mit Unterstützung der Deutschen Forschungsgemeinschaft durchgeführt. Wir danken Herrn Dr. R. KNupPeN für die Überlassung der radioaktiven Steroide, Frl. Mechthild BrckManN für ihre wertvolle Mitarbeit und Herrn Prof. Dr. E. J. Plotz, Direktor der UniversitätsFrauenklinik Bonn, für sein stetes Intercsse.

\section{Literatur}

1. PATt, V. und J. Breuer, diese Z. 7, 108 (1969). - 2. Breuer, J., W. Meusers und H. Breuer, diese Z. 6, 163 (1968). 3. Folin, O. und V. Ciocalteu, J. biol. Chemistry 73, 627 (1927). - 4. Werthessen, N. T., C. F. BAKer und B. BorCi, Science Washington 107, 64 (1948). - 5. VAN DER MOLEN, H. J. und D. Groen, Acta endocr. Khvn 58, 419 (1968). - 6. Brown,
B. T., W. S. Golder und S. E. Wrighr, Austral. J. Exper. Biol. 39, 345 (1961). - 7. Hormone Assays and their Clinical Application, hsg. Loraine, J. A. und E. T. Bell, Livingstone, Edinburgh \& London (1966). - 8. KLopper, A., Obstetr. Survey Baltimore 23, 813 (1968). - 9. RyaN, K. J., R. MEIGS und Z. Petro, Amer. J. Obstetr. Gynec. 69, 676 (1966).

Prof. Dr. H. Breuer

Inst. f. Klin. Biochemie 53 Bonn-Venusberg 